\title{
Digitale Technologien und die Wiederentdeckung der relativen Einzelkostenrechnung nach Riebel
}

\author{
Steffen Menze*
}

Die Digitalisierung und Big Data bringen einen wegweisenden Technologiefortschritt mit sich, mit dem im Finanzbereich eine hohe Erwartungshaltung verbunden ist: Insbesondere im Controlling werden effiziente Prozesse, qualitativ hochwertige Daten sowie eine bessere Unterstützung bei der Entscheidungsfindung erwartet. Das Controlling steht nun vor der Herausforderung, mit welchen Methoden und digitalen Technologien diese Erwartungen erfüllt werden können.

Dabei lohnt sich einen Blick auf das im Controlling wohl etablierteste Informationssystem: Die Kostenrechnung. Sie war in der Vergangenheit immer wieder als zu universell und statisch für die heutige Zeit kritisiert worden. Das ist per se auch nicht verwunderlich, da sowohl die gängigen Voll- als auch Teilkostenrechnungssysteme vor mehr als einem halben Jahrhundert vor allem für die industrielle (Massen-)Fertigung von Einzelprodukten innerhalb einer Periode (i. d. R. ein Kalenderjahr) konzipiert wurden. Verbraucher verlangen heute allerdings nach individuellen und dynamisch wechselnden Produktangeboten. Zudem werden immer häufiger Produkte und Dienstleistungen kombiniert miteinander angeboten. Eine solche Entwicklung der Märkte bringt die tradierten Kostenrechnungssysteme an ihre Grenzen. Insbesondere produktbegleitende und mitunter periodenübergreifende Dienstleistungen (z. B. Versicherungen, lebenslange Garantieleistungen und Hotlines beim Neukauf eines PKW) können sie nur wenig differenziert über pauschale Zuschläge auf die Höhe der Einzelkosten erfassen (Weber 2021: 225ff).

\footnotetext{
Steffen Menze I Leiter Controlling / Rechnungswesen I GESIS Gesellschaft für Informationssysteme mbH (ein Unternehmen der Salzgitter Gruppe)

Der Beitrag beruht auf dem Arbeitspapier „Kostenrechnerische Erfassung von Verbundprodukten mittels Bezugsobjekthierarchien"von Steffen Menze, GESIS Gesellschaft für Informationssysteme mbH (ein Unternehmen der Salzgitter Gruppe) | Dr. Fabian Flämmer, dennree GmbH | Dr. Frank Tiefenbeck, BearingPoint | Prof. Dr. Barbara E. Weißenberger, Heinrich-Heine-Universität Düsseldorf, das für das Projekt „Verbundprodukte“ im Arbeitskreis Internes Rechnungswesen der SchmalenbachGesellschaft für Betriebswirtschaft e.V. erstellt wurde. Alle hier genannten Thesen geben die Meinung des Autors wieder.
} 
Durch den Einsatz digitaler Technologien allein können diese systemimmanenten Kritikpunkte nicht gelöst werden. Fruchtbringend ist allerdings, diese mit dem in der betrieblichen Praxis in den letzten Jahrzehnten in Vergessenheit geratenem Kostenrechnungssystem zu verbinden, das von seinem Grundaufbau und Gedankengut her genau diese Informationsbedarfe adressiert: Die relative Einzelkosten- und Deckungsbeitragsrechnung (kurz: relative Einzelkostenrechnung), deren Anwendung in der Vergangenheit häufig u. a. aufgrund von technologischen Grenzen unterblieb. Daher ist es nur konsequent, die relative Einzelkostenrechnung im Lichte neuer digitaler Technologien zu betrachten.

\section{Funktionsweise der relativen Einzelkostenrechnung}

Die relative Einzelkostenrechnung war von Paul Riebel bereits in den 1950er Jahren speziell für die praktische Anwendung in Unternehmen mit Kuppelproduktion konzipiert worden. Dabei handelt es sich um Produkte, die durch die Aufspaltung eines Rohstoffs (z.B. Rohöl in Benzin, Diesel, Heiz- und Schweröl sowie Bitumen) entstehen. Damit entfallen auf die Kostenträger anders als z.B. bei zusammenbauender Fertigung nur geringe Einzelkosten (z.B. aus späteren Fertigungsschritten) und die Kalkulationsverfahren wie z.B. die Zuschlagskalkulation sind nicht sinnvoll anwendbar.

Der von Riebel hierfür entwickelte Lösungsansatz lässt sich auf alle Produktionsverfahren übertragen, indem im System der relativen Einzelkostenrechnung parallel zueinander aufgebaute Bezugsobjekthierarchien definiert werden, die unternehmensindividuell nach regelmäßig wiederkehrenden Gesichtspunkten sowie situativen Entscheidungsproblemen strukturiert sind. Den einzelnen Ästen dieser Hierarchien werden sodann die für sie relevanten Erlös- und Kostenpositionen zugeordnet. Alle Kostenpositionen, die an der untersten Ebene einer solchen Hierarchie zugeordnet werden, stellen dabei Einzelkosten dar. Alle weiteren Kosten in höheren Hierarchieebenen werden bezogen auf diese Hierarchieebene als Gemeinkosten, bezogen auf ihre eigene Ebene wiederum als Einzelkosten angesehen (,relative“ Einzelkosten). Die Unterscheidung von Erlösen folgt der gleichen Vorgehensweise (Riebel 1994: 35ff).

Die Zuordnung der einzelnen Erlös- und Kostenpositionen in Form von Deckungsbeiträgen erfolgt nach dem Identitätsprinzip, d.h. Kosten und Leistungen dürfen nur dann einem Bezugsobjekt auf einer Hierarchieebene zugerechnet werden, wenn sie auf identische Entscheidungen zurückgehen. Die umsetzungsbezogene Logik ist dann recht einfach: Von der untersten Ebene einer Hierarchie ausgehend, wird geprüft, ob Kosten oder Erlöse einem Ast eindeutig zugeordnet werden können. Ist das nicht der Fall, ist die nächsthöhere Ebene zu prüfen und so fort. Das Vorgehen gewinnt jedoch an Komplexität, wenn mehrere Hierarchien existieren, denn dann sind Erlös- und Kostenpositionen auch mehrfach zuzuordnen. Das Gehalt eines Produktmanagers kann demnach Einzelkosten einer nach Produkten und Gemeinkosten einer nach regionalen Gesichtspunkten aufgestellten Bezugsobjekthierarchie sein.

Aus dem Identitätsprinzip heraus resultiert ein von Riebel konzipierter entscheidungsorientierter Erlös- bzw. Kostenbegriff. Demnach stellen im Unternehmensalltag getroffene Entscheidungen den Ursprung sämtlicher 
Erlöse bzw. Kosten dar (Riebel 1994: 427). Da die monetären Auswirkungen der getroffenen Entscheidungen einem Entscheidungsobjekt bzw. einem Ast in den Bezugsobjekthierarchien eindeutig - über das Identitätsprinzip - zugeordnet werden können, wird auf eine pauschalierte oder geschlüsselte Erfassung von Erlösen wie Kosten vollständig verzichtet.
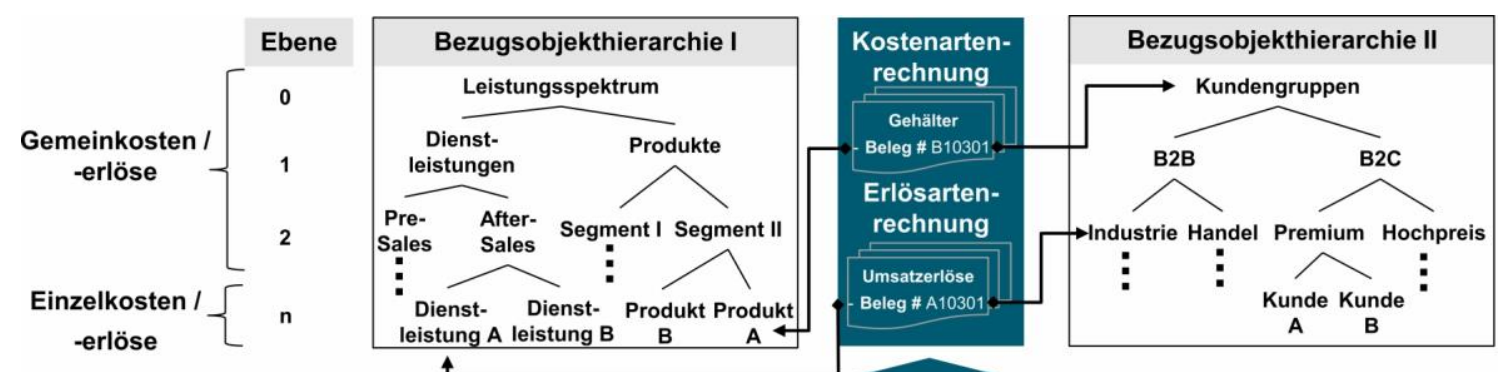

\begin{tabular}{|c|l|c|c|c|c|c|c|}
\hline \multicolumn{9}{|c|}{ Zweckneutrale Datenbasis } \\
\hline Beleg \# & \multicolumn{1}{|c|}{ Kosten-I Erlösart } & Menge & Gesellschaft & Hierarchie I & Hierarchie II & $\ldots$ & Wert (Mio. $\boldsymbol{\epsilon}$ ) \\
\hline B10301 & 211 Gehälter & 1 & 30 & & & & -100 \\
\hline A10301 & 103 Umsatzerlöse & 10.000 & 10 & & & & 700 \\
\hline B10302 & 210 Administration & 1 & 20 & & & & -500 \\
\hline
\end{tabular}
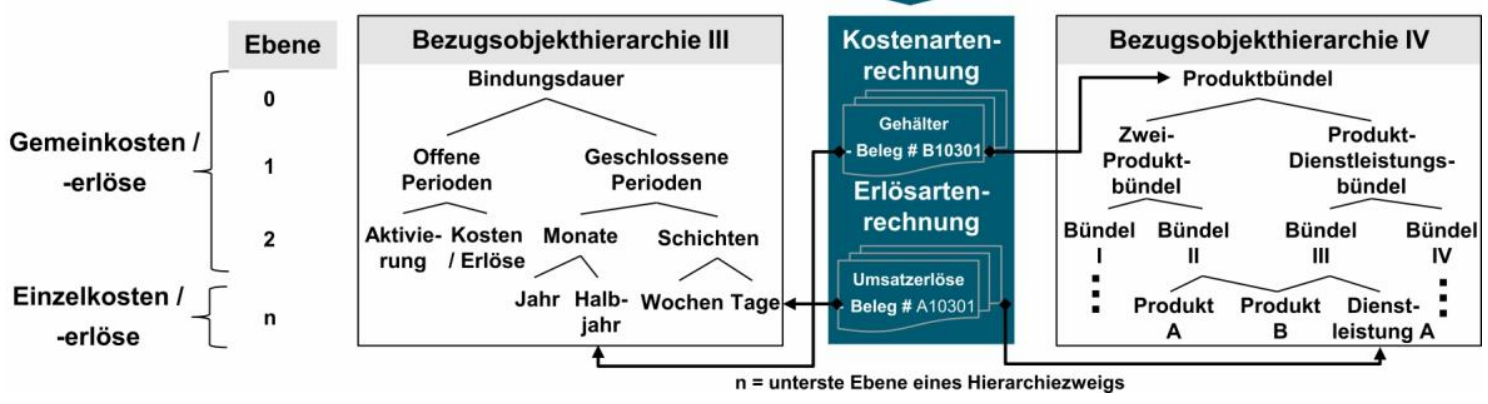

Abb. 1: Funktionsweise der relativen Einzelkostenrechnung

\section{Warum hat sich die relative Einzelkostenrechnung bisher nicht durchgesetzt?}

Die relative Einzelkostenrechnung ist über Jahrzehnte hinweg in der Wissenschaft vor allem wegen ihres überhöhten Absolutheitsanspruchs sehr kontrovers diskutiert worden (Weber und Weißenberger 1997: 284f). Hinzu kamen die Herausforderungen in der betriebspraktischen Umsetzung, denn die Implementierung einer relativen Einzelkostenrechnung erfordert einen hohen Aufwand bei der Datenerfassung: Jeder einzelne Buchungsbeleg - einschließlich der Belegpositionen - ist zu prüfen und in der Regel mehreren Hierarchien zuzuordnen. Selbst bei kleinen Unternehmen betrifft das nicht selten mehrere Tausend Belege pro Monat. Erleichterungen lassen sich nur durch regelbasierte Zuordnungen erzielen, die allerdings recht pauschal, fehleranfällig und betreuungsintensiv in der Anwendung sind. Dem sehr differenzierten Anspruch der relativen Einzelkostenrechnung und dem entscheidungsorientierten Kostenbegriff kann damit nicht entsprochen werden (Riebel 1994: 451ff). 


\section{Nutzung von Machine Learning zur Datenerfassung}

Die heute zur Verfügung stehenden Ansätze des Machine Learning bieten allerdings weiterführende Möglichkeiten an, um insbesondere die Datenerfassung effizienter und differenzierter zu gestalten. So kann die Datenerfassung durch einen Algorithmus erfolgen, der darauf „trainiert“ wird, die Zuordnung von Buchungsbelegpositionen zu unterschiedlichen Bezugsobjekthierarchien selbstständig vorzunehmen. Dabei fließen neu gewonnene (statistische) Erkenntnisse immer wieder in das Modell ein, um dieses ständig zu verbessern. Insofern kann eine datengetriebene Belegpositionszuordnung vorgenommen werden, die selbstlernend und weniger betreuungsintensiv ist, da nicht sämtliche Regeln der Belegzuordnung ex ante bekannt sein müssen.

\section{Performante Datenbanktechnologien zur Datenspeicherung}

Die Datenhaltung in ERP-Systemen verändert sich grundlegend. Waren in der Vergangenheit Daten an vielen verschiedenen Stellen einer relationalen Datenbank gespeichert, stellt etwa die dem ERP-System SAP S/4HANA zugrundeliegende In-Memory-Datenbanktechnologie lediglich einen einzelnen zentralen Datenspeicherort - das „Universal Journal“ bereit. Ein „Single source of truth“ wird somit allein aus technischer Sicht sichergestellt und redundante Daten vermieden. Schnellere Zugriffsgeschwindigkeiten durch bessere Hardware und eine neue Form der Datenspeicherung (,,spaltenorientierte Datenhaltung“) erhöhen zudem die Verarbeitungsgeschwindigkeit deutlich. Immer größere Datenmengen können folglich verarbeitet werden („Big Data“), ohne dass es einer Verdichtung der Daten bedarf. Gleichfalls können entlang der Datenbasis frei definierbare Merkmale - wie etwa Bezugsobjekthierarchien - hinzugefügt werden, ohne dass das Datenmodell adjustiert werden muss. Der Aufbau der Datenbank entspricht folglich den Anforderungen an eine zweckneutrale Datenbasis, wie sie die relative Einzelkostenrechnung fordert (Weber 2021: 297f sowie Abb. 1).

\section{Prozesseffizienz wird gesteigert}

Während in herkömmlichen Kostenrechnungssystemen eine sukzessive Verrechnungsfolge zwischen Kostenarten-, Kostenstellen- und Kostenträgerrechnung vorgesehen ist, werden die drei Grundelemente einer Kostenrechnung in der relativen Einzelkostenrechnung integriert betrachtet. Verrechnungsprozesse innerhalb der Kostenstellen- und anschließend in die Kostenträgerrechnung sind daher obsolet. Damit findet aus prozessualer Sicht ein Front-Loading klassischer Monatsabschlusstätigkeiten im Controlling statt. Standen die Ergebnisse der Kostenrechnung bis dato zeitlich verzögert zur Verfügung, kann nunmehr - zumindest konzeptionell - ein Live-Reporting generiert werden. Die Zuordnung von Buchungsbelegen zu Bezugsobjekthierarchien direkt bei der Verbuchung macht dies möglich. Die gewonnenen (statistischen) Erkennt- 
nisse aus einer datengetriebenen Belegzuordnung können auch als Grundlage für fruchtbare Diskussionen in weiteren Prozessen dienen: Wird bspw. ein auf dem Entscheidungsbaumverfahren basierender Algorithmus verwendet, baut der Algorithmus anhand der im Buchungsbeleg mitgeführten Informationen ein entsprechendes (mehrstufiges) Regelwerk mit Zuordnungskriterien auf, anhand dessen die Belegzuordnung erfolgt. Der auf diese Art und Weise generierte Entscheidungsbaum entspricht im Ergebnis einem (Wert-)Treibermodell, das etwa in Planungsprozessen für Umsatz- und Kostenprognosen herangezogen werden kann.

\section{Hohe Datenqualität kann erreicht werden}

Ein wesentlicher Vorteil durch den Einsatz digitaler Technologien ist, dass Auswertungen bis auf Einzelbelegebene jeder Zeit durchgeführt werden können, da eine Datenverdichtung obsolet ist. Das Rechnen mit Durchschnittswerten rückt damit in den Hintergrund. Stattdessen kann selbst bei komplexen Fragestellungen mit spezifischen Werten bis hinunter zum Ursprungsbeleg agiert werden. Die gewonnene Granularität kann noch durch die Einführung von mehreren Bezugsobjekthierarchien verstärkt werden, die einen mehrdimensionalen Blick auf die Daten erlauben. Differenzierte Analysen werden somit möglich (Weber 2021: 261). Das Arbeiten mit Bezugsobjekthierarchien bietet zudem eine hohe Flexibilität. So können umfangreiche Re-Organisationen oder neue Reporting-Anforderungen mit verhältnismäßig geringem Aufwand durch Hinzufügen einer neuen Hierarchie abgebildet werden. Bestehende Hierarchien können zudem beibehalten werden.

\section{Entscheidungsunterstützung wird forciert}

Das Arbeiten mit Bezugsobjekthierarchien i. V. m. den Konzepten des Identitätsprinzips und dem relativen Deckungsbeitragsbegriff können dazu beitragen, Problemfelder traditioneller Kostenrechnungssysteme zu überwinden und die Entscheidungsunterstützung durch das Controlling zu forcieren. So können kombiniert miteinander angebotene Produkte und Dienstleistungen mithilfe von Bezugsobjekthierarchien prinzipiell abgebildet und zum Zweck der Entscheidungsfundierung und -nachverfolgung aufbereitet werden. Digitale Technologien können vor allem dazu beitragen, solche Verbundwirkungen überhaupt erst zu identifizieren. Der Einsatz von Cluster-Analysen kann bei der Identifizierung unterstützen, aber auch wertvolle Hinweise für die Zusammensetzung neuer, bis dato nicht offerierter Verbundprodukte, generieren. Mehr noch: Durch den strikten Verzicht auf Schlüsselungen sowie Pauschalierungen von Erlösen und Kosten findet in der relativen Einzelkostenrechnung auch kein Verwässerungseffekt dieser Positionen statt. Daher kann das Datenkonstrukt grundsätzlich auch für (mathematische) Optimierungsrechnungen herangezogen werden (Mertens et al. 1977: 78f). Denkbar ist beispielsweise eine Optimierung des Produktportfolios unter weiterer Einbeziehung einer nach regionalen Gesichtspunkten strukturierten Bezugsobjekthierarchie. Des Weiteren können Hierarchien für ad-hoc-Fragestellungen aufgebaut bzw. herangezogen werden. Bei Desinvestitionsvorhaben kann eine solche Vor- 
gehensweise dabei unterstützen, die tatsächlich relevanten Erlöse und Kosten des herauszulösenden Bereichs zu identifizieren (Weber und Weißenberger 1997: 297).

\section{Fazit}

Digitale Technologien geben dem Controlling wesentliche Impulse. Neue Anwendungsfelder werden möglich, die in der Vergangenheit allein durch technologische und methodische Grenzen verschlossen waren. Von besonderer Bedeutung ist dabei das Zusammenspiel aus Technologie und Methodik. Nur eine konsequente Verbindung von beidem ermöglicht es letztlich, die Erwartungshaltung an die Digitalisierung im Finanzbereich zu erfüllen und einen Mehrwert für das Unternehmen zu generieren. Theoretisch fundierte Konzepte wie die relative Einzelkostenrechnung können diese unterstützen. Gelingt dies, kann die Kostenrechnung von einem universellen und statischen Informationssystem zu einer individuell gestaltbaren und dynamisch agierenden ,datengetriebenen Kostenrechnung، ausgebaut werden.

\section{Literaturverzeichnis}

Mertens, P., K. Hansen, G. Rackelmann. 1977. Selektionsentscheidungen im Rechnungswesen - Überlegungen zu computergestützten Kosteninformationssystemen. Die Betriebswirtschaft 37(1): 77-88.

Riebel, P. 1994. Einzelkosten- und Deckungsbeitragsrechnung. Grundfragen einer markt- und entscheidungsorientierten Unternehmensrechnung. 7. Aufl., Wiesbaden.

Weber, J., B.E. Weißenberger. 1997. Relative Einzelkosten- und Deckungsbeitragsrechnung: a critical evaluation of Riebel's approach. Management Accounting Research 8(3): 277-298.

Weber, J. 2021. Zukunftsfähige Kostenrechnung in der Unternehmenssteuerung. Analysen, Strategien, Lösungen. Freiburg et al. 